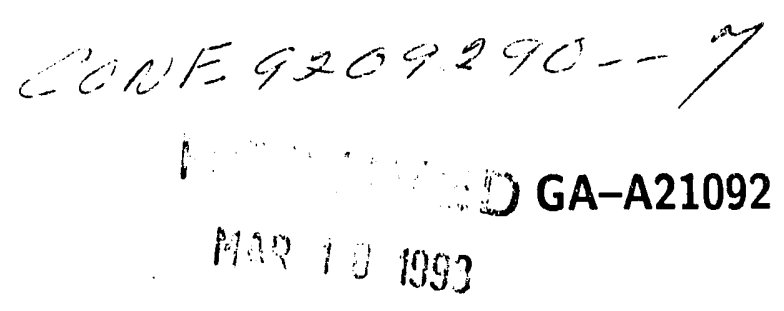

\title{
CONFINEMENT AND STABILITY OF VH-MODE DISCHARGES IN THE DIII-D TOKAMAK
}

by

T.S. TAYLOR, T.H. OSBORNE, K.H. BURRELL, T.N. CARLSTROM, V.S. CHAN, M.S. CHU, J.C. DeBOO, E.J. DOYLE, C.M. GREENFIELD, R.J. GROEBNER, C.L. HSIEH, G.L. JACKSON, R. JAMES, L.L. LAO, E.A. LAZARUS, S.I. LIPPMAN, T.W. PETRIE, C.L. RETTIG, H. St. JOHN, D.P. SCHISSEL, R.D. STAMBAUGH, E.J. STRAIT, A.D. TURNBULL, W.P. WEST, J. WINTER, D. WROBLEWSKI, and THE DIII-D TEAM

OCTOBER 1992 


\section{DISCLAIMER}

This report was prepared as an account of work sponsored by an agency of the United States Government. Neither the United States Government nor any agency thereof, nor any of their employees, makes any warranty, express or implied, or assumes any legal liability or responsibility for the accuracy, completeneas, or usefulness of any information, apparatus, product, or process disclosed, or represents that its use would not infringe privately owned rights. Reference herein to any specific commercial product, process, or service by trade name, trademark, manufacturer, or otherwise, does not necessarily constitute or imply its endorsement, recommendation, or favoring by the United States Government or any agency thereof. The views and opinions of authors expressed herein do not neceasarily state or reflect those of the United States Government or any agency thereof. 


\section{CONFINEMENT AND STABILITY OF VH-MODE DISCHARGES IN THE DIII-D TOKAMAK}

by

T.S. TAYLOR, T.H. OSBORNE, K.H. BURRELL, T.N. CARLSTROM, V.S. CHAN, M.S. CHU, J.C. DeBOO, E.J. DOYLE,* C.M. GREENFIELD, R.J. GROEBNER, C.L. HSIEH, G.L. JACKSON, R. JAMES,** L.L. LAO, E.A. LAZARUS, ${ }^{* * *}$ S.I. LIPPMAN, T.W. PETRIE, C.L. RETTIG,* H. St. JOHN, D.P. SCHISSEL, R.D. STAMBAUGH, E.J. STRAIT, A.D. TURNBULL, W.P. WEST, J. WINTER, **** D. WROBLEWSKI, ${ }^{* *}$ and THE DIII-D TEAM

This is a preprint of a paper to be presented at the 14th International Conference on Plasma Physics and Controlled Nuclear Fusion, September 30-October 7, 1992, Würzburg, F.R.G., and to be printed in the Proceedings.

\section{Work supported by U.S. Department of Energy Contracts DE-AC03-89ER51114, DE-AC05-840R21400, and W-7405-ENG-48}

\footnotetext{
*Permanent Address: University of California at Los Angeles, U.S.A.

**Permanent Address: Lawrence Livermore National Laboratory, U.S.A.

***Permanent Address: Oak Ridge National Laboratory, U.S.A.

****Permanent Address: Kernforschungsanlage Jülich GmbH, F.R.G.
}

GENERAL ATOMICS PROJECT 3466 OCTOBER 1992

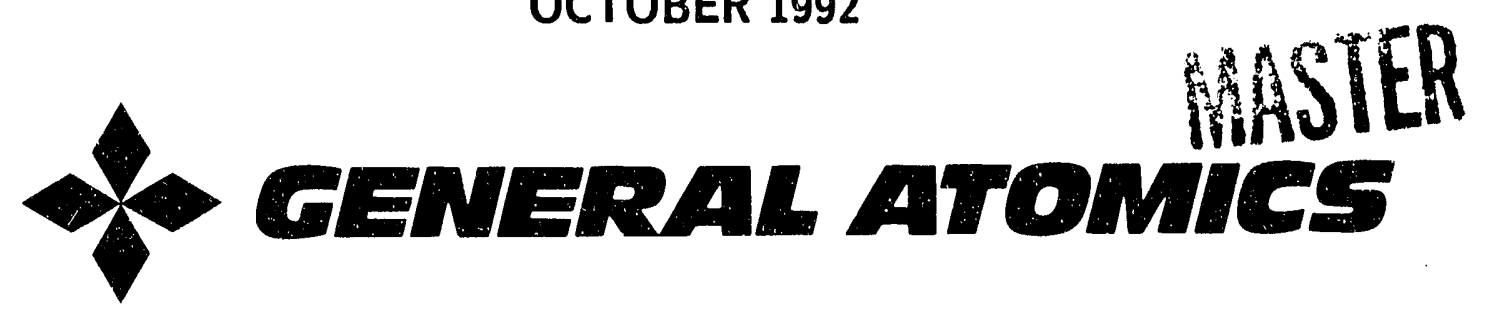


IAEA CN-56/A-3-1

\title{
CONFINEMENT AND STABILITY OF \\ VH-MODE DISCHARGES IN THE DIII-D TOKAMAK
}

\begin{abstract}
A regime of very high confinement (VH-mode) has been observed in neutral beam-heated deuterium discharges in the DIII-D tokamak with thermal energy confinement times up to $\approx 3.6$ times that predicted by the ITER-89P $\mathrm{L}$-mode scaling and 2 times that predicted by ELM-free H-mode thermal confinement scalings. This high confinement has led to increased plasma performance, $n_{D}(0) \mathrm{T}_{i}(0) \tau_{E}=2 \times 10^{20} \mathrm{~m}^{-3} \mathrm{keV}$ sec with $I_{p}=1.6 \mathrm{MA}, \mathrm{B}_{T}=2.1 \mathrm{~T}$, $Z_{\text {eff }} \leq 2$.

Detailed transport analysis shows a correspondence between the large decrease in thermal diffusivity in the region $0.75 \leq \rho \leq 0.9$ and the development of a strong shear in the radial electric field in the same region. This suggests that stabilization of turbulence by sheared $E \times B$ flow is responsible for the improved confinement in VH-mode. A substantial fraction of the edge plasma entering the second regime of stability may also contribute to the increase in confinement. The duration of the VH-mode phase has been lengthened by feedback controlling the input power to limit plasma beta.
\end{abstract}

\section{INTRODUCTION}

The viability of a tokamak as a fusion reactor depends on the simultaneous achievement of good confinement and high beta. We report here the observation of a regime of high confinement (VH-mode) $[1,2]$ in DIII-D, with global thermal energy confinement times up to twice those predicted by the JET/DIII-D ELMfree $\mathrm{H}$-mode scaling [3], corresponding to global energy confinement times up to 3.6 times those predicted by the ITER-89P L-mode scaling [4]. These high confinement times are observed even close to the predicted MHD beta limit.

In this paper, we will describe the operational conditions needed for VH-mode, including the role of discharge shape. Transport in VH-mode and H-mode discharges will be compared, and possible mechanisms for the improved confinement will be discussed. The MHID instability which usually terminates the VH-phase will be described, along with possible means of avoiding the instability and extending the duration of this mode of operation. Recent experimental work has concentrated on experiments at 1.6 MA, 2.1 T to evaluate the cause of the improved confinement, and we report those results here. 


\section{VH-MODE DISCHARGE CHARACTERISTICS AND DISCHARGE EVOLUTION}

The VH-mode regime of high confinement was observed in DIII-D following boronization of the vessel wall in May 1991 [2]. Discharges with distinctive $\mathrm{VH}$-mode characteristics have since been obtained over the range of $1.0 \mathrm{MA}$ $\leq I_{p} \leq 2 \mathrm{MA}, 4 \mathrm{MW} \leq P_{\mathrm{NBI}} \leq 16 \mathrm{MW}$, and $1.7 \leq \mathrm{B}_{T} \leq 2.1 \mathrm{~T}$. The high confinement times are observed at high values of normalized beta, $\beta_{N}=\beta_{T} /(\mathrm{I} / \mathrm{aB})$ (\% T-m/MA) $2 \leq \beta_{N} \leq 3.5$, and high $\beta_{T}, \beta_{T} \lesssim 4 \%$. This high confinement at high beta has led to increased plasma performance: $n_{d}(0) \mathrm{T}_{i}(0) \tau_{E}=2 \times 10^{20} \mathrm{~m}^{-3}$ $\mathrm{keV}$ sec with $I_{p}=1.6 \mathrm{MA}, \mathrm{B}_{T}=2.1 \mathrm{~T}, Z_{\text {eff }}<2$.

The most distinctive feature of the $\mathrm{VH}$-mode discharge is the increase in the thermal energy confinement time during the ELM-free phase of the beam heating pulse. $\mathrm{VH}$-mode develops out of the $\mathrm{H}$-mode during the ELM-free period. The temporal evolution of a VH-mode discharge and an $\mathrm{H}$-mode discharge with a long ELM-free period are compared in Fig. 1. Both are deuterium discharges with the same level of constant, deuterium neutral beam injection power $(9 \mathrm{MW}$ ), high triangularity, $\delta \approx 0.85$, and double-null divertor (DND) configuration. An L-H transition occurs shortly after the start of beam injection, as is seen by the drop in the divertor $\mathrm{D}_{\alpha}$ emission. After the initial rise in $\tau$ following the $\mathrm{L}-\mathrm{H}$ transition, the thermal energy confinement time of the $\mathrm{H}$-mode decreases slightly during the ELM-free phase as the loss power $\left(P_{\text {LOSS }}=P_{\mathrm{ABS}}-d W / d t\right)$ increases, consistent with $\mathrm{P}_{\mathrm{LOSS}}^{-1 / 2}$ confinement scalings. In contrast, the thermal energy confinement time in the $\mathrm{VH}$-mode continues to increase during the ELM-free phase, although the loss power, plasma energy, and $\beta$ continue to increase. In many discharges, there is an apparent "transition" over tens of msec, during which there is substantial change in the interior toroidal rotation and radial electric field and the confinement increases more rapidly. In discharges where the transition is identifiable, the transition occurs at $\beta_{p} \gtrsim 1$. With a constant input power greater than $8 \mathrm{MW}$, the plasma beta and the energy confinement time continue to increase until the VH-mode phase is terminated by a global MHD event near the predicted beta limit: in the case shown $\beta_{N}$ reaches 3.1. The peak in the energy confinement time just before the VH-mode termination is more than twice that predicted by the JET/DIII-D ELM-free H-mode thermal energy confinement scaling, $\tau_{\mathrm{JET} / \mathrm{DII}-\mathrm{D}}=0.106 I_{p}^{1.03}(\mathrm{MA}) P^{-0.46}(\mathrm{MW}) R^{1.48}(\mathrm{~m})$. Following the termination of the VH-phase, the discharge reverts to an ELMing $\mathrm{H}$-mode, with a confinement equal to that predicted by $\mathrm{H}$-mode scaling relations.

There are several additional characteristics and operational requirements of VH-mode. VH-mode is obtained during the long ELM-free phase of the discharge and the high confinement regime is almost always terminated with the beginning of ELMs. VH-mode is obtained with low radiated power, low ohmic target density $\left(\approx 3 \times 10^{19} \mathrm{~m}^{-3}\right.$ at $\left.1.6 \mathrm{MA}\right)$, low recycling, and high triangularity, conditions previously recognized as important in obtaining long ELM-free periods. Discharges with higher target densities and higher recycling have a shorter ELM-free period; and as a consequence of the earlier onset of ELMs, have a 

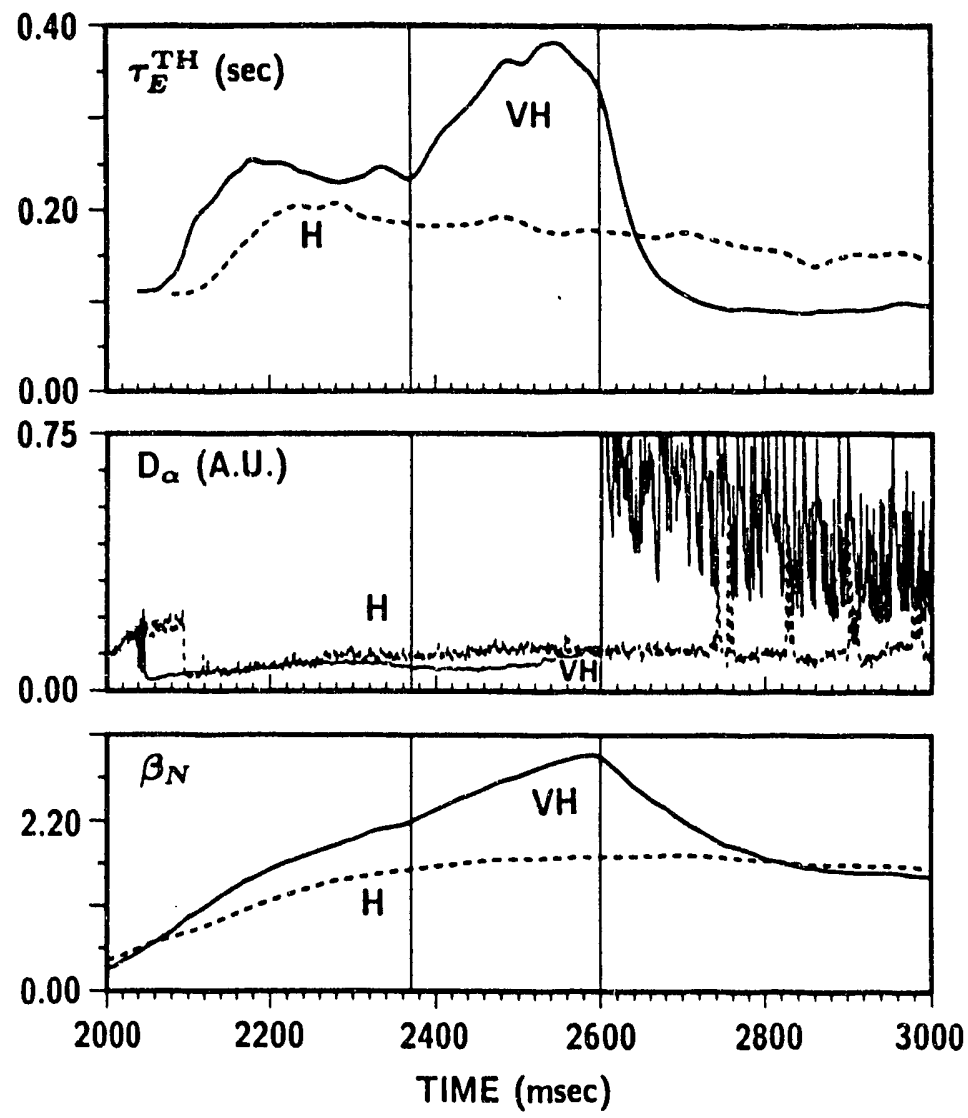

Fig. 1. Temporal evolution of $\mathrm{VH}$-mode and ELM-free $\mathrm{H}$-mode. Solid curves are for 1.6 MA, double-null divertor, $\mathrm{VH}$-mode; dashed curves are for 2.0 MA double-null divertor ELM-free $H$-mode. $\tau_{E}^{\mathrm{TH}}$, thermal energy confinement time; $D_{\alpha}$, deuterium $\alpha$ emission from the divertor region; $\beta_{N}$, normalized beta. Data used in the calculation of confinement times are averaged over $40 \mathrm{msec}$.

lower peak energy confinement time. Even following boronization, five to eight minutes duration of helium glow discharge cleaning between tokamak discharges is required to maintain the low target density and low recycling in order to reliably obtain VH-mode discharges. It is felt that one of the key consequences of boronization is the ability to produce moderate to high current discharges with high beam power and low target density. Prior to boronization, locked modes and/or radiative collapse limited the performance of $\mathrm{H}$-mode discharges [2].

The radiated power in $\mathrm{VH}$-mode discharges is significantly reduced over that of $\mathrm{H}$-modes obtained prior to boronization. In $\mathrm{H}$-mode discharges prior to boronization, the radiated power increases throughout the ELM-free phase, and at high current $\left(I_{p} \gtrsim 2 \mathrm{MA}\right)$, the ELM-free phase is often terminated when the radiated power reaches $\approx 70 \%$ of the input power. The radiation profile of these 
discharges is nearly flat, with the high central radiation caused by the accumulation of metal impurities. The radiated power in the VH-mode discharges is typically less than $30 \%$ of the input power and the radiation emission is strongly localized near the plasma edge. This low radiated power is correlated with a significant reduction in metal impurity radiation; a consequence of improved edge plasma shielding, reducing the impurity influx in VH-mode discharges [5], and the boron coverage of the metal vessel wall.

The enhanced confinement in the $\mathrm{VH}$-mode may be associated with a large current density near the plasma edge. The high edge current density may play a significant role in establishing the improved confinement or it may be a consequence of the high confinement. This edge current is deduced from the MHD equilibrium reconstruction which derives from the measured pressure profile, the external magnetics, and the internal field pitch angle calculated from the motional Stark effect measurement [6]. Transport analysis using measured thermal plasma profiles shows this edge current to be bootstrap driven current. In discharges where the edge current density was suppressed by an applied negative EMF (a negative current ramp), the peak energy confinement time wa. significantly reduced compared to non-ramped discharges. This observation is in contrast to other current ramp experiments in L-mode limiter plasmas where negative current ramps and the increase in the internal inductance resulted in an increase in the confinement compared to non-ramped discharges at the same value of current [7].

\section{GLOBAL ENERGY CONFINEMENT}

The energy confinement of VH-mode discharges is nearly twice that of other ELM-free discharges, see Fig. 1. This increase in the thermal energy confinement taken at the peak value during a discharge is compared to the JET/ DIII-D ELM-free H-mode thermal confinement scaling in Fig. 2: the solid curve represents 2 times $\tau_{\text {JET/DIII-D }}$ scaling. Many VH-mode discharges reach two times $\tau_{\text {JET/DIII-D. }}$. We have not yet completed enough systematic single parameter scans to perform a statistically defensible regression analysis, however, the data are consistent with $\tau_{E} \propto I_{p} P_{\text {LOSS }}^{-0.5}$ [2].

The highest peak energy confinement in DIII-D discharges is observed at high triangularity in $\mathrm{VH}$-mode. The confinement of single-null divertor (SND) discharges with triangularity, $\delta \approx 0.7$, is only slightly less than that of the DND discharges, and the discharge evolution is very similar. At lower triangule :ity (below $\delta \approx 0.5$ ), the peak energy confinement is further reduced, the ELM-free phase terminates smoothly with the commencement of small ELMs, and we do not observe what we call the VH-mode. The dependence of the peak energy confinement on the triangularity is shown in Fig. 3. Even following boronization, during ELM- free SND discharges with $\delta \approx 0.3, \tau_{E} \lesssim 1.2 \tau_{\text {JET/DIII-L }}$. 


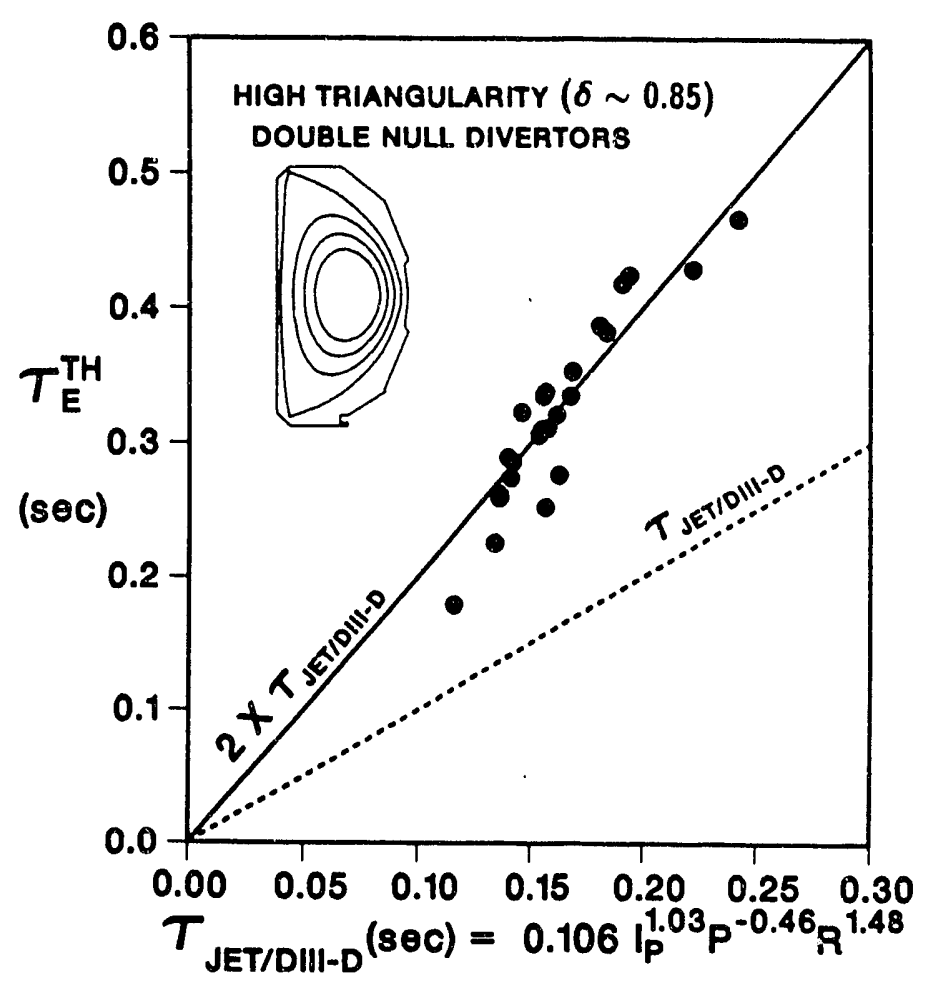

Fig. 2. Comparison of VH-mode thermal confinement for double-null divertors $(\delta \approx 0.85)$ to JET/DIII-D ELM-free H-mode scaling. $\tau_{E}^{\mathrm{TH}}=W_{\mathrm{TH}} /\left[P_{\mathrm{ABS}}-(d W / d t)\right], W_{\mathrm{TH}}=$ $W_{\text {MHD }}$ - W FAST. $W_{\text {FAST }}$ is fast ion energy content based on calculated slowing down time and injected neutral beam power. $1 \leq I_{p} \leq 2 \mathrm{MA}, 4 \leq P_{\text {LOSS }} \leq 8 \mathrm{MW}$.

\section{PROFILES AND TRANSPORT}

The high energy confinement of the VH-mode is seen to coincide with the broadening of the steep density gradient and temperature gradient region for both electrons and ions in the outer portion of the plasma. A comparison of the measured profiles for 1.6 MA double-null divertor $\mathrm{L}$-mode, $\mathrm{H}$-mode, and VH-mode discharges is shown in Fig. 4. The shapes of the electron temperature and ion temperature profiles in the central region of the $\mathrm{L}$-mode and $\mathrm{H}$-mode discharges are similar. The $\mathrm{H}$-mode has a noticeable increase in the temperature and density near the boundary resulting in large gradients outside $\rho \approx 0.9$. These strong gradients outside $\rho \approx 0.9$ give what some refer to as an edge "pedestal" in the plasma energy. In VH-mode the steep gradients in the electron temperature, the ion temperature and the electron density all extend further into the plasma, typically to $\rho \approx 0.8$. There is a very noticeable increase in the temperature gradient near $\rho \approx 0.8$ from $\mathrm{H}$-mode to $\mathrm{VH}$-mode. Note that 


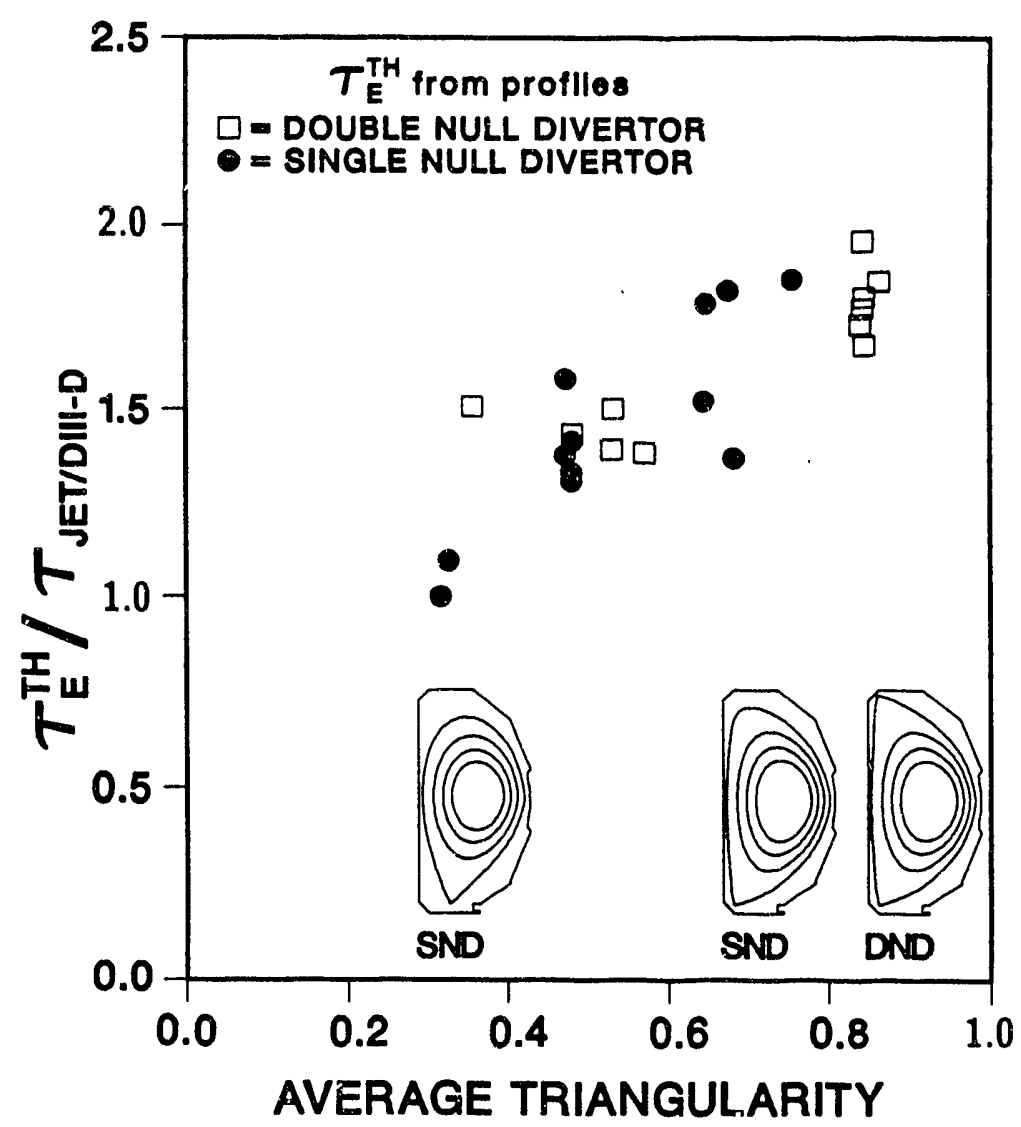

Fig. 3. Dependence of peak energy confinement times on discharge triangularity: open squares are single-null divertors, closed circles are double-null divertors.

the electron density in VH-rnode is quite high, $\bar{n}_{e} \gtrsim 6 \times 10^{19} \mathrm{~m}^{-3}$, and as a consequence of the high $n_{e}$, the ion and electron temperatures are nearly equal and the fast ion energy content is less than $10 \%$ of the total plasma energy. The ion and the fast ion content can be higher in lower current and lower density discharges. The $Z_{\text {eff }}$ profile for both $\mathrm{H}$-mode and $\mathrm{VH}$-mode is slightly hollow with $Z_{\text {eff }} \approx 1$ near the center and increasing to $\approx 1.5$ to 2 near the boundary (approximately equal $\mathrm{B}$ and $\mathrm{C}$ contributions).

To better understand the cause of the improved confinement, the local transport characteristics of the discharges are compared. In general, ELM-free $\mathrm{H}$-mode and $\mathrm{VH}$-mode are continuously evolving and time dependent transport analysis is required. The $\mathrm{L}$-mode discharges are quasi-stationary and a single time independent transport analysis suffices. MHD equilibria are reconstructed from the poloidal magnetic measurements, the diamagnetic measurement, the poloidal field pitch from Motional Stark [6] measurements, and the kinetic pressure. The kinetic pressure profile is constructed from the measured $T_{e}, T_{i}$, $n_{e}, Z_{\text {eff, }}$ and the calculated fast ion content. The transport coefficients are 


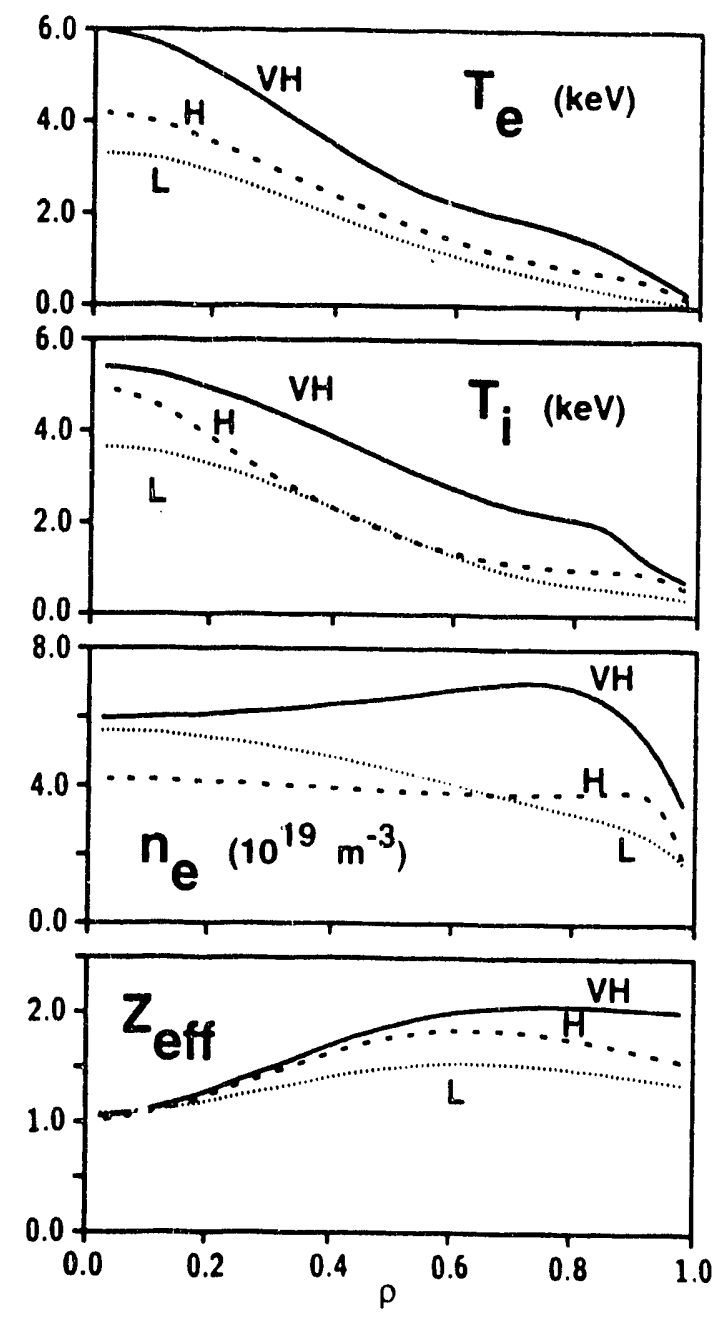

Fig. 4. Comparison of the kinetic profiles for $\mathrm{VH}$-mode (solid), $\mathrm{H}$-mode (dashed), L-mode (dotted); $T_{e}$, electron temperature, from Thomson scattering [8]; $T_{i}$, ion temperature, from charge exchange recombination spectroscopy [9]; $n_{e}$, electron density, from Thomson scattering and two infrared interferometer ct ords; $Z_{\text {eff, effective ion charge, deduced from }}$ bremsstrahlung emission [10].

calculated in the couje ONETWO [11], using the reconstructed equilibria, the measured profiles $\left(\mathrm{T}_{e}, \mathrm{~T}_{i}, n_{e}, Z_{\mathrm{eff}}, P_{\mathrm{rad}}\right)$, and the calculated beam deposition and slowing down as input. Because $T_{e}$ and $T_{i}$ are quite close and the electron density is high, there is a large uncertainty in the ion electron transfer term. Thus, a single fluid thermal diffusivity is used, $\chi_{\text {eff }}=-q_{\mathrm{eff}} /\left(n_{\mathrm{e}} \nabla \mathrm{T}_{e}+n_{i} \nabla \mathrm{T}_{i}\right)$, where $q_{\text {eft }}$ is the heat flux. 
The improvement in confinement from $\mathrm{L}$-mode to $\mathrm{H}$-mode is a consequence of the development of a transport barrier at the edge of the discharge, $\rho \gtrsim$ 0.85 . The further improvement in confinement from $\mathrm{H}$-mode to $\mathrm{VH}$-mode can be viewed as a broadening of this transport barrier into $\rho \approx 0.75$. The $\mathrm{L}$-mode discharge exhibits a steeply rising thermal diffusivity towards the plasma boundary. In $\mathrm{H}$-mode, the diffusivity is reduced over most of the discharge, including the bulk, but most noticeably in the edge, beyond $\rho \approx 0.85$, Fig. 5 .

Comparing $\mathrm{VH}$-mode to $\mathrm{H}$-mode, $\chi_{\text {eff }}$ further reduces in the outer region of the plasma, especially in the region of $0.75 \lesssim \rho \lesssim 0.9$. Inside $\rho \approx 0.6$ the change in the thermal diffusivity is within the error bars. These changes in the

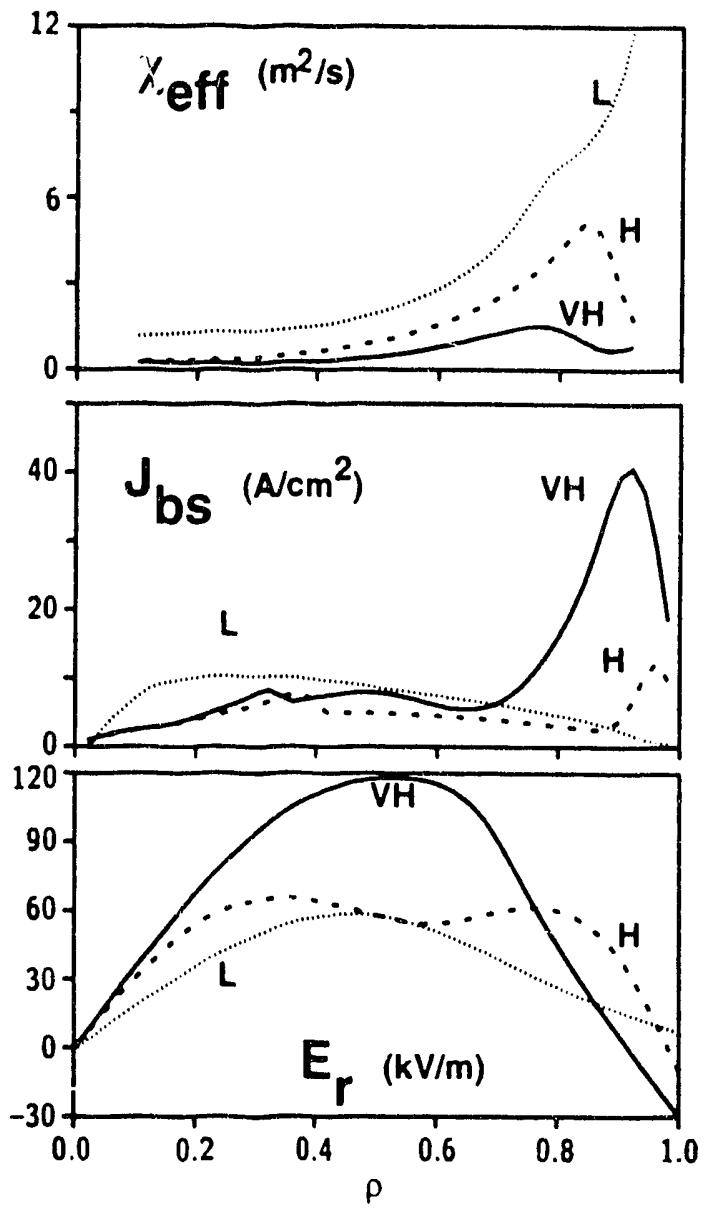

Fig. 5. Comparison of transport results and radial electric field for VH-mode (solid),

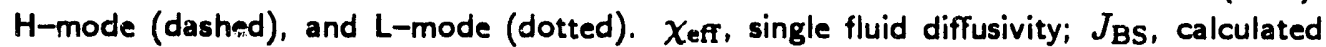
bootstrap current; $E_{r}$, radial electric field. 
thermal diffusivity can be inferred directly from the profiles shown in Fig. 4. In $\mathrm{L}$-mode the gradients are all quite weak near the boundary, $\rho \gtrsim 0.8$. In $\mathrm{H}$-mode, a steep gradient develops in $\mathrm{T}_{e}, \mathrm{~T}_{i}$, and $n_{e}$ outside $\rho \approx 0.85$ to 0.9 . In $\mathrm{VH}$-mode, the steep gradient in all the profiles extends into $\rho \approx 0.75$ to 0.8 . A number of 1.6 MA H-modes and $\mathrm{VH}$-modes have been analyzed, and although the details of the thermal diffusivity profile change, the general features in Fig. 5 are reproduced. It is the transport in the region between $0.75 \lesssim \rho \leqq 0.9$ that is reduced from $\mathrm{H}$-mode to $\mathrm{VH}$-mode and is largely responsible for the improved confinement.

The calculated bootstrap current density and the radial electric field shear are also shown in Fig. 5. The bootstrap current in the well developed VH-mode peaks at $\rho \approx 0.85$. The radial electric field is calculated from the rotationinduced Doppler shift in the CER CVI emission [9], and the CVI pressure gradient: $E_{r}=v \times B+\left(1 / n_{I} \rho_{I}\right) \nabla P_{I}$. The H-mode develops a shear in the radial electric field in the outer region, primarily at $\rho \gtrsim 0.9$. In the $\mathrm{VH}$-mode this radial electric field extends inward to $\rho \approx 0.7$.

\section{VH-MOI TERMINATION}

The VH-mode phase is often terminated by a global MHD event near the expected beta limit. An example of $\mathrm{VH}$-mode termination is shown in Fig. 6. The event is initiated by an $n=5 \mathrm{MHD}$ mode rotating in the electron diamagnetic drift direction. The mode grows on a timescale of 20 to $50 \mu \mathrm{sec}$ [Fig. 6(b)]. Mode numbers $n=3$ to 5 are typically observed in other discharges and in some cases an $n=1$ mode which grows on a timescale of tens of msec is observed on the magnetics and the central SXR emission leading up to the termination. Rapid drops in edge SXR emission and a rise in $D_{\alpha}$ begin during the growth of the $n=5$ mode and extend to the central SXR chord within $300 \mu \mathrm{sec}$. From ${ }^{2}$ Thomson scattering, a drop in the electron temperature as much as a factor of 2 is sometimes observed within $20 \mathrm{msec}$ of the appearance of the $n=1$ mode and the $\mathrm{D}_{\alpha}$ spike. Using experimental equilibria at peak beta, ideal kink stability calculations with the code GATO [11] show ideal modes with $n=2,3,4$ to be unstable and $n=1$ near marginal stability, consistent with the observed modes.

This instability and termination of the VH-mode phase tend to become more violent (global with greater prompt energy loss) as $\beta$ increases. In discharges with lower input power and lower $\beta$, the MHD event appears more localized to the edge. The MHD mode at the event in the lower $\beta$ case is still typically $2<n<5$, but is a lower amplitude mode. The similarity between the MHD event for a hard termination and a soft termination indicates that the underlying physics may be the same. The global MHD event occurs at normalized beta values of $2.5<\beta_{N}<3.5$, in agreement with the expected beta limit of divertor discharges with high edge current density [9]. The coupling to the $n=1$ 


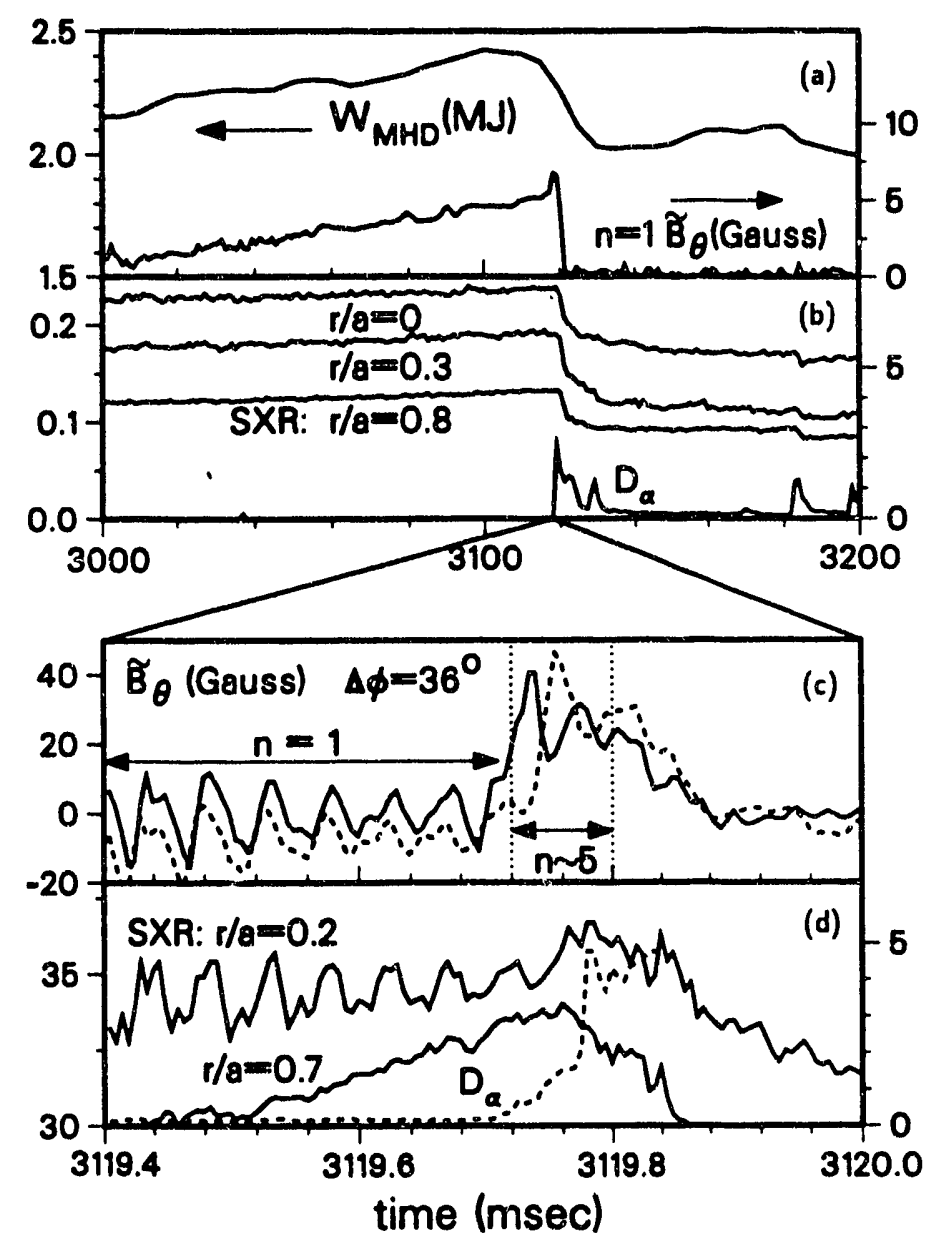

Fig. 6. VH-mode termination. (a) Plasma energy ( $W_{\mathrm{MHD}}$ ), amplitude of $n=1$ magnetic fluctuations, $\tilde{B}_{\theta}$, (b) soft $\mathrm{X}$-ray emission from three chords, $\mathrm{D}_{\alpha}$ emission from the divertor, (c) expanded trace of magnetic probe signals, $\tilde{B}_{\theta}$. (d) expanded trace of soft $X$-ray emission and $D_{\alpha}$ emission from the divertor.

mode may result in the global character of the termination and the substantial prompt energy loss. The avoidance of the $n=1$ either through current profile control or maintaining lower beta might prevent the abrupt global termination.

\section{PROGRESS TOWARD LONG-PULSE ENHANCED CONFINEMENT}

Two techniques have been found which allow operation for longer duration with confinement substantially above that of ELM-free $\mathrm{H}$-mode confinement as predicted by JET/DIII-D scaling. The first of these is to feedback control the 
neutral beam input power to maintain the plasma beta below the theoretically predicted beta limit. Using the digital control system [14], the bearn power is feedback controlled to hold the diamagnetic flux constant by changing the chopping frec $c_{1}$ lency and/or number of ion sources. With this feedback control scheme, the diamagnetic flux and $\beta_{N}$ are held constant resilting in VH-mode discharges with ELM-free periods in excess of $1 \mathrm{sec}$, and $\tau_{E}^{\mathrm{TF}} \approx 2 \tau_{\mathrm{JET} / \mathrm{DIII}-\mathrm{D}}$ for up to $0.6 \mathrm{sec}$. The temporal evolution of such a discharge is shown in Fig. $7(a-c)$.
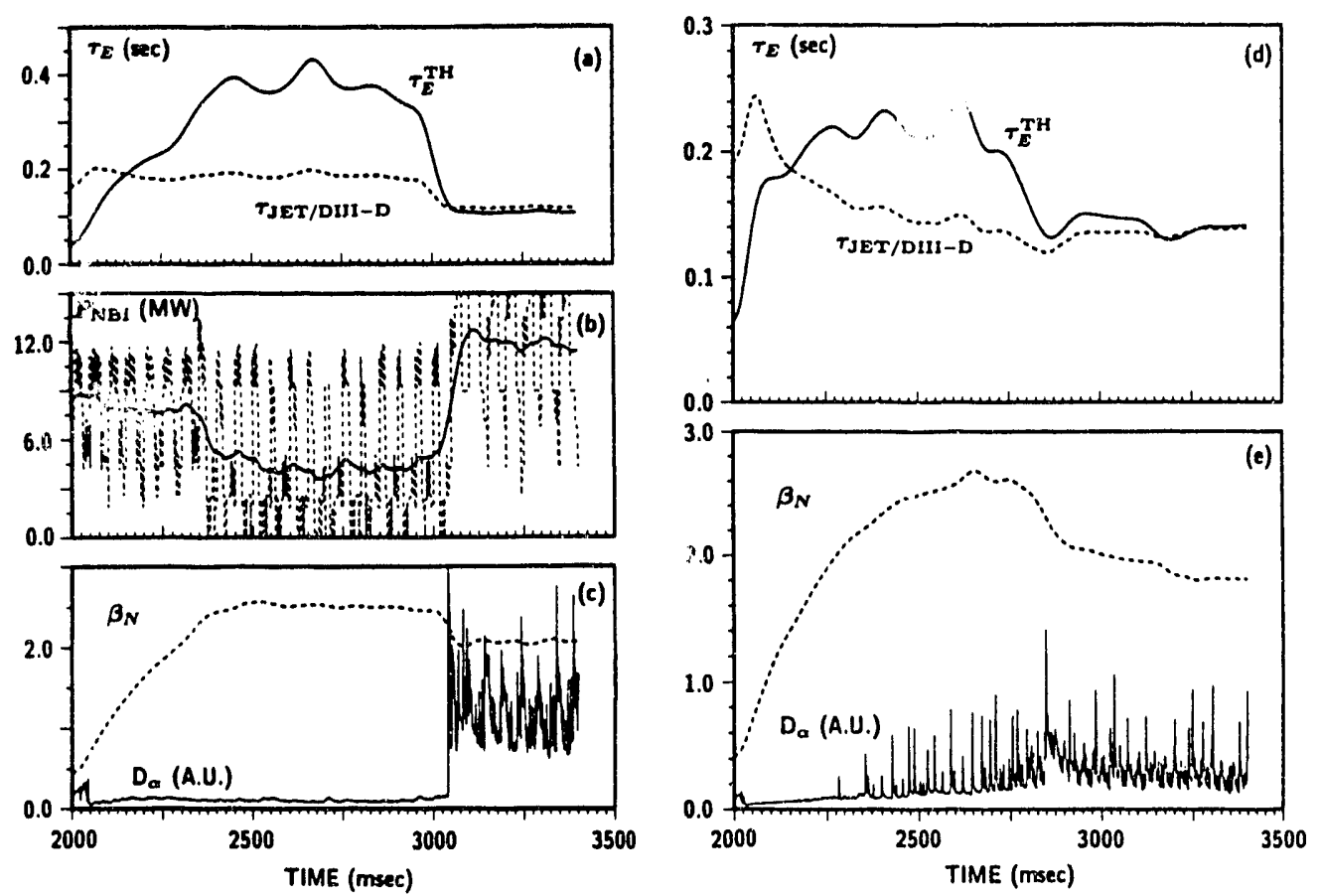

Fig. 7. Long pulse enhanced confinement discharges: $(a-c)$ Feedback on the input power; (d-e) ELMing discharge: $\tau_{E}^{\mathrm{TH}}$ (solid), thermal energy confinement time; $\tau_{\text {JET/DIII-D }}$ (dashed), predicted energy confinement from JET/DIII-D scaling; $P_{\text {NBI }}$, injected neutral beam power (dashed), average of injected power (solid); $\beta_{N}$ (dashed), normalized beta; $D_{\alpha}$ (solid), deuterium $\alpha$-emission from the divertor region.

In a high triangularity SND discharge with moderate input power, substantially improved confinement is observed in the presence of small and frequent ELMs [Fig. 7(d,e)]. In this discharge, the confinement time develops similar to other VH-mode discharges following the $\mathrm{L}-\mathrm{H}$ transition at $2030 \mathrm{msec}$. At $2300 \mathrm{msec}$ small and frequent ELMs commence as can be seen on the divertor $\mathrm{D}_{\alpha}$ emission. The energy confinement time is greater than 1.5 times that predicted by JET/DIII-D scaling.

Although true steady state VH-mode discharges have not yet been obtained, these two examples indicate the possibility. It is noted that for some 
time after the discovery of H-mode, ELMs often terminated the period of good confinement, anci only later was it discovered that the ELMs could be used as a rrieans to obtain near steady state $\mathrm{H}$-modes. We anticipate with further evaluation and experiment that we will be able to produce steady state $\mathrm{VH}$-mode discharges in the future.

\section{DISCUSSION}

A plausible explanation for the increased confinement in VH-mode is the expansion of the region of sheared $E \times B$ stabilization of microturbulence. The correspondence between the development (temporally and spatially) of the increased shear in the radial electric field and the decrease in the calculated diffusivity supports this model. This is a well-developed model for explaining the improvement in edge confinement in $\mathrm{H}$-mode plasmas $[15,16]$. In the $\mathrm{H}$-mode the shear in the radial electric field is strongest in the region beyond $\rho \approx 0.85$, and likewise, the reduction in transport from $\mathrm{L}$-mode to $\mathrm{H}$-mode is largest in this region. In a typical evolution of a VH-mode discharge, from $100 \mathrm{msec}$ fol lowing the $\mathrm{L}-\mathrm{H}$ transition to $\approx 400 \mathrm{msec}$ following the transition, the energy confinement time and the calculated profile of thermal diffusivity and radial electric field remain nearly unchanged ( $\tau_{E}$ may increase slightly). As the energy confinement begins to increase more rapidly, the radial electric field at $\rho \approx 0.6$ increases rapidly and the electric field shear increases. At this same time the relative density fluctuations, $\tilde{n}_{e} / n_{e}$, measured by infrared scattering decrease [17].

The change in the radial electric field occurs primarily in a few tens of msec and leads the change in the temperature and density profiles. The region of strong radial electric field shear in VH-mode extends from $0.65 \leqslant \rho \leq 1$ compared to $0.85 \lesssim \rho \leq 1$ for $\mathrm{H}$-mode discharges. This expansion of the strong shear region corresponds to the observed expansion of the transport barrier in $\mathrm{VH}$-mode and the substantial reduction in thermal diffusivity in the region $0.75 \lesssim \rho \lesssim 0.9$ of $\mathrm{VH}$-mode.

Another possible explanation for the improvement in confinement in $\mathrm{VH}$-mode is expansion of the region of second stability to ballooning modes near the plasma boundary. There are three mechanisms by which second regime access could lead to reduced transport: (1) Stabilization of ideal and/or resistive ballooning modes [18] in the plasma edge could lead to the improved confinement. The edge temperatures in VH-mode are substantially higher than in $\mathrm{H}$-mode and the edge collisionality is lower; and resistive ballooning modes are therefore likely to be more easily stabilized. Second regime stability to ideal modes alone is not singularly sufficient for the improvement in edge transport since there are a number of DIII-D H-mode discharges with edge region second regime access, but with little or no improvement in confinement. (2) Second 
regime access of the plasma edge alters the character and increases the stability of ELMs [19]: the absence of ELMs is required for the best confinement discharges. (3) Local shear reversal in the unfavorable curvature region, high $\beta_{p}$, and strong shaping are favorable for drift reversal of trapped particles and stabilization of trapped particle modes, and could lead to reduced transport in low collisionality plasmas. Second stability regime access $\mathrm{i}_{i}$ ear the plasma edge is a consequence of the large edge current density in moderate $q(q \gtrsim 4)$ shaped plasmas. Using simulated equilibria, the volume of the plasma in the second stability regime $n \in$ ar the plasma boundary is shown to increase with increasing edge current density [2].

A high edge bootstrap current is a natural consequence of the operational conditions for which VH-mode is obtained. Low target density, low recycling, and significant input power lead to low edge density, high edge temperature, and low edge collisionality. The low edge collisionality and the high pressure gradient observed in $\mathrm{H}$-mode discharges lead to high edge bootstrap current.

\section{CONCLUSIONS}

Discharges in DIII-D with substantially improved confinement have been obtained over a large range of parameters The exact cause of the improved transport is still being investigated, but stabilization of microturbulence by sheared $E \times B$ flows seems to be a good model. Steady-state VH-modes with density control by ELMs might be possible. With improved stability limits, the very high confinement observed in VH-mode discharges might provide a path to a more compact, economically attractive tokamak reactor and could provide a significant margin for ignition in ITER.

\section{ACKNOWLEDGMENTS}

The authors would like to thank S. Thompson for computational support. This is a report of work sponsored by the U.S. Department of Energy under Contracts DE-AC03-89ER51114, DE-AC05-84OR21400, and W-7405-ENG-48.

\section{REFERENCES}

[1] JACKSON, G.L., et al., Phys. Rev. Lett. 67 (1991) 3098.

[2] JACKSON, G.L., et al., Phys. Fluids B 4 (1992) 2181.

[3] SCHISSEL, D.P., et al., Nucl. Fusion 31 (1991) 73.

[4] YUSHMANOV, P.N., et al., Nucl. Fusion 30 (1990) 1999.

[5] LIPPMANN, S.I., etal., "Impurity Penetration and Transport During VH-mode on DIII-D," to be published in J. Nucl. Mater. 
[6] LAO, L.L., et al., Nucl. Fusion 30 (1990) 1035.

[7] LAO, L.L., et al., IAEA-CN-56/A-7-19, this volume.

[8] CARLSTROM, T.N., et al., "Design and Operation of the Multipulse Thomson Scattering Diagnostic on DIII-D," Rev. Sci. Instrum., in press.

[9] GOHIL, P.H., et al., Proc. 16th IEEE/NPSS Symposium on Fusion Technology (IEEE, San Diego, 1991), Vol. II, p. 1199.

[10] SCHISSEL, D.P., et al., Phys. Fluids 31 (1988) 12.

[11] PFEIFFER, W.W., et al., General Atomics Report GA-A17178 (1980).

[12] BERNARD, L.C., et al., Comput. Phys. Commun. 24 (1981) 377.

[13] TAYLOR, T.S., et al., Plasma Physics and Controlled Nuclear Fusion Research 1990 (Proc. 13th Int. Conf. Washington, DC, 1990), Vol. 1, LAEA, Vienna, (1991) 177.

[14] FERRON, J.R., et al., Proc. 14th IEEE/NPS Symposium on Fusion Engineering (IEEE, New York, 1989), Vol. 2, p. 761.

[15] BURRELL, K.H., etal., Phys. Fluids B 2 (1990) 1405.

[16] BURRELL, K.H., et al., "Physics of the L-Mode to H-Mode Transition in Tokamaks," Plasma Physics and Controlled Fusion 34, in press.

[17] DOYLE, E.J., et al., IAEA-CN-56/A-4-1, this volume.

[18] BISHOP, C.M., Nucl. Fusion 26 (1986) 1063.

[19] OZEKI, T., et al., Nucl. Fusion 30 (1990) 1425.

[20] GLASSER, A.H., et al., Phys. Fluids 17 (1974) 181.

[21] MILLER, R.L., etal., Comments Plasma Physics Controlled Fusion 12 (989) 125. 

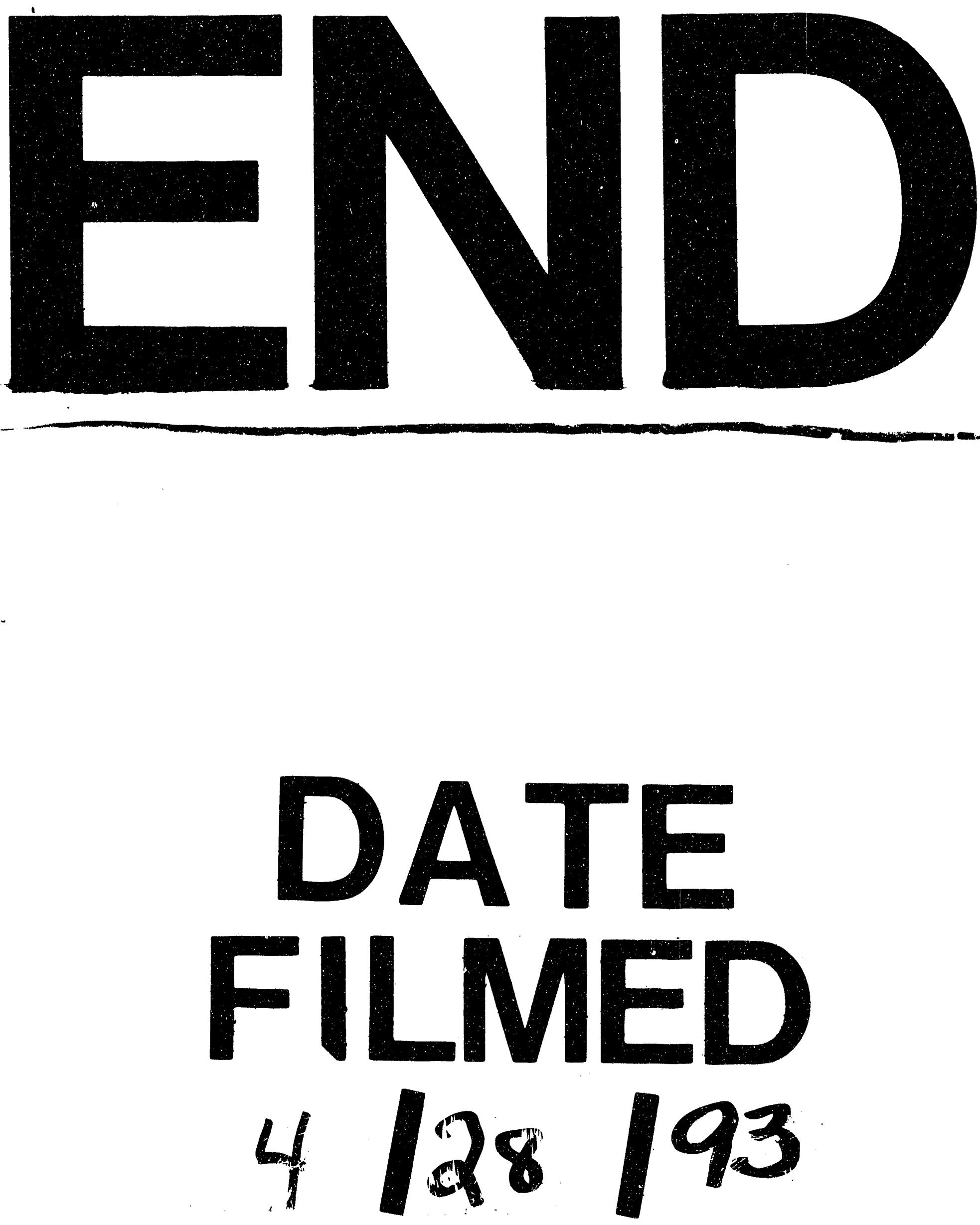
